Geometry \& Topology Monographs

Volume 3: Invitation to higher local fields

Part II, section 8, pages 281-292

\title{
8. Higher local skew fields
}

\author{
Alexander Zheglov
}

$n$-dimensional local skew fields are a natural generalization of $n$-dimensional local fields. The latter have numerous applications to problems of algebraic geometry, both arithmetical and geometrical, as it is shown in this volume. From this viewpoint, it would be reasonable to restrict oneself to commutative fields only. Nevertheless, already in class field theory one meets non-commutative rings which are skew fields finite-dimensional over their center $K$. For example, $K$ is a (commutative) local field and the skew field represents elements of the Brauer group of the field $K$ (see also an example below). In [Pa] A.N. Parshin pointed out another class of non-commutative local fields arising in differential equations and showed that these skew fields possess many features of commutative fields. He defined a skew field of formal pseudodifferential operators in $n$ variables and studied some of their properties. He raised a problem of classifying non-commutative local skew fields.

In this section we treat the case of $n=2$ and list a number of results, in particular a classification of certain types of 2-dimensional local skew fields.

\subsection{Basic definitions}

Definition. A skew field $K$ is called a complete discrete valuation skew field if $K$ is complete with respect to a discrete valuation (the residue skew field is not necessarily commutative). A field $K$ is called an $n$-dimensional local skew field if there are skew fields $K=K_{n}, K_{n-1}, \ldots, K_{0}$ such that each $K_{i}$ for $i>0$ is a complete discrete valuation skew field with residue skew field $K_{i-1}$.

\section{Examples.}

(1) Let $k$ be a field. Formal pseudo-differential operators over $k((X))$ form a 2dimensional local skew field $K=k((X))\left(\left(\partial_{X}^{-1}\right)\right), \partial_{X} X=X \partial_{X}+1$. If $\operatorname{char}(k)=$ 0 we get an example of a skew field which is an infinite dimensional vector space over its centre. 
(2) Let $L$ be a local field of equal characteristic (of any dimension). Then an element of $\operatorname{Br}(L)$ is an example of a skew field which is finite dimensional over its centre.

From now on let $K$ be a two-dimensional local skew field. Let $t_{2}$ be a generator of $\mathcal{M}_{K_{2}}$ and $t_{1}^{\prime}$ be a generator of $\mathcal{M}_{K_{1}}$. If $t_{1} \in K$ is a lifting of $t_{1}^{\prime}$ then $t_{1}, t_{2}$ is called a system of local parameters of $K$. We denote by $v_{K_{2}}$ and $v_{K_{1}}$ the (surjective) discrete valuations of $K_{2}$ and $K_{1}$ associated with $t_{2}$ and $t_{1}^{\prime}$.

Definition. A two-dimensional local skew field $K$ is said to split if there is a section of the homomorphism $\mathcal{O}_{K_{2}} \rightarrow K_{1}$ where $\mathcal{O}_{K_{2}}$ is the ring of integers of $K_{2}$.

Example (N. Dubrovin). Let $\mathbb{Q}((u))\langle x, y\rangle$ be a free associative algebra over $\mathbb{Q}((u))$ with generators $x, y$. Let $I=\langle[x,[x, y]],[y,[x, y]]\rangle$. Then the quotient

$$
A=\mathbb{Q}((u))\langle x, y\rangle / I
$$

is a $\mathbb{Q}$-algebra which has no non-trivial zero divisors, and in which $z=[x, y]+I$ is a central element. Any element of $A$ can be uniquely represented in the form

$$
f_{0}+f_{1} z+\ldots+f_{m} z^{m}
$$

where $f_{0}, \ldots, f_{m}$ are polynomials in the variables $x, y$.

One can define a discrete valuation $w$ on $A$ such that $w(x)=w(y)=w(\mathbb{Q}((u)))=0$, $w([x, y])=1, w(a)=k$ if $a=f_{k} z^{k}+\ldots+f_{m} z^{m}, f_{k} \neq 0$. The skew field $B$ of fractions of $A$ has a discrete valuation $v$ which is a unique extension of $w$. The completion of $B$ with respect to $v$ is a two-dimensional local skew field which does not split (for details see [Zh, Lemma 9]).

Definition. Assume that $K_{1}$ is a field. The homomorphism

$$
\varphi_{0}: K^{*} \rightarrow \operatorname{Int}(K), \quad \varphi_{0}(x)(y)=x^{-1} y x
$$

induces a homomorphism $\varphi: K_{2}^{*} / \mathcal{O}_{K_{2}}^{*} \rightarrow \operatorname{Aut}\left(K_{1}\right)$. The canonical automorphism of $K_{1}$ is $\alpha=\varphi\left(t_{2}\right)$ where $t_{2}$ is an arbitrary prime element of $K_{2}$.

Definition. Two two-dimensional local skew fields $K$ and $K^{\prime}$ are isomorphic if there is an isomorphism $K \rightarrow K^{\prime}$ which maps $\mathcal{O}_{K}$ onto $\mathcal{O}_{K^{\prime}}, \mathcal{M}_{K}$ onto $\mathcal{M}_{K^{\prime}}$ and $\mathcal{O}_{K_{1}}$ onto $\mathcal{O}_{K_{1}^{\prime}}, \mathcal{M}_{K_{1}}$ onto $\mathcal{M}_{K_{1}^{\prime}}$. 


\subsection{Canonical automorphisms of infinite order}

\section{Theorem.}

(1) Let $K$ be a two-dimensional local skew field. If $\alpha^{n} \neq$ id for all $n \geqslant 1$ then char $\left(K_{2}\right)=\operatorname{char}\left(K_{1}\right), K$ splits and $K$ is isomorphic to a two-dimensional local skew field $K_{1}\left(\left(t_{2}\right)\right)$ where $t_{2} a=\alpha(a) t_{2}$ for all $a \in K_{1}$.

(2) Let $K, K^{\prime}$ be two-dimensional local skew fields and let $K_{1}, K_{1}^{\prime}$ be fields. Let $\alpha^{n} \neq \mathrm{id}, \alpha^{\prime n} \neq \mathrm{id}$ for all $n \geqslant 1$. Then $K$ is isomorphic to $K^{\prime}$ if and only if there is an isomorphism $f: K_{1} \rightarrow K_{1}^{\prime}$ such that $\alpha=f^{-1} \alpha^{\prime} f$ where $\alpha, \alpha^{\prime}$ are the canonical automorphisms of $K_{1}$ and $K_{1}^{\prime}$.

\section{Remarks.}

1. This theorem is true for any higher local skew field.

2. There are examples (similar to Dubrovin's example) of local skew fields which do not split and in which $\alpha^{n}=$ id for some positive integer $n$.

Proof. (2) follows from (1). We sketch the proof of (1). For details see [Zh, Th.1].

If $\operatorname{char}(K) \neq \operatorname{char}\left(K_{1}\right)$ then $\operatorname{char}\left(K_{1}\right)=p>0$. Hence $v(p)=r>0$. Then for any element $t \in K$ with $v(t)=0$ we have $p t p^{-1} \equiv \alpha^{r}(\bar{t}) \bmod \mathcal{M}_{K}$ where $\bar{t}$ is the image of $t$ in $K_{1}$. But on the other hand, $p t=t p$, a contradiction.

Let $F$ be the prime field in $K$. Since $\operatorname{char}(K)=\operatorname{char}\left(K_{1}\right)$ the field $F$ is a subring of $\mathcal{O}=\mathcal{O}_{K_{2}}$. One can easily show that there exists an element $c \in K_{1}$ such that $\alpha^{n}(c) \neq c$ for every $n \geqslant 1$ [Zh, Lemma 5].

Then any lifting $c^{\prime}$ in $\mathcal{O}$ of $c$ is transcendental over $F$. Hence we can embed the field $F\left(c^{\prime}\right)$ in $\mathcal{O}$. Let $\bar{L}$ be a maximal field extension of $F\left(c^{\prime}\right)$ which can be embedded in $\mathcal{O}$. Denote by $L$ its image in $\mathcal{O}$. Take $\bar{a} \in K_{1} \backslash \bar{L}$. We claim that there exists a lifting $a^{\prime} \in \mathcal{O}$ of $\bar{a}$ such that $a^{\prime}$ commutes with every element in $L$. To prove this fact we use the completeness of $\mathcal{O}$ in the following argument.

Take any lifting $a$ in $\mathcal{O}$ of $\bar{a}$. For every element $x \in L$ we have $a x a^{-1} \equiv$ $x \bmod \mathcal{M}_{K}$. If $t_{2}$ is a prime element of $K_{2}$ we can write

$$
a x a^{-1}=x+\delta_{1}(x) t_{2}
$$

where $\delta_{1}(x) \in \mathcal{O}$. The map $\overline{\delta_{1}}: L \ni x \rightarrow \overline{\delta_{1}(x)} \in K_{1}$ is an $\alpha$-derivation, i.e.

$$
\overline{\delta_{1}}(e f)=\overline{\delta_{1}}(e) \alpha(f)+e \overline{\delta_{1}}(f)
$$

for all $e, f \in L$. Take an element $h$ such that $\alpha(h) \neq h$, then $\overline{\delta_{1}}(a)=g \alpha(a)-a g$ where $g=\overline{\delta_{1}}(h) /(\alpha(h)-h)$. Therefore there is $a_{1} \in K_{1}$ such that

$$
\left(1+a_{1} t_{2}\right) a x a^{-1}\left(1+a_{1} t_{2}\right)^{-1} \equiv x \bmod \mathcal{M}_{K}^{2} .
$$

By induction we can find an element $a^{\prime}=\ldots \cdot\left(1+a_{1} t_{2}\right) a$ such that $a^{\prime} x a^{\prime-1}=x$. 
Now, if $\bar{a}$ is not algebraic over $\bar{L}$, then for its lifting $a^{\prime} \in \mathcal{O}$ which commutes with $L$ we would deduce that $L\left(a^{\prime}\right)$ is a field extension of $F\left(c^{\prime}\right)$ which can be embedded in $\mathcal{O}$, which contradicts the maximality of $L$.

Hence $\bar{a}$ is algebraic and separable over $\bar{L}$. Using a generalization of Hensel's Lemma [Zh, Prop.4] we can find a lifting $a^{\prime}$ of $\bar{a}$ such that $a^{\prime}$ commutes with elements of $L$ and $a^{\prime}$ is algebraic over $L$, which again leads to a contradiction.

Finally let $\bar{a}$ be purely inseparable over $\bar{L}, \bar{a}^{p^{k}}=\bar{x}, x \in L$. Let $a^{\prime}$ be its lifting which commutes with every element of $L$. Then $a^{p^{k}}-x$ commutes with every element of $L$. If $v_{K}\left(a^{\prime p^{k}}-x\right)=r \neq \infty$ then similarly to the beginning of this proof we deduce that the image of $\left(a^{\prime p^{k}}-x\right) c\left(a^{p^{k}}-x\right)^{-1}$ in $K_{1}$ is equal to $\alpha^{r}(c)$ (which is distinct from $c$ ), a contradiction. Therefore, $a^{p^{k}}=x$ and the field $L\left(a^{\prime}\right)$ is a field extension of $F\left(c^{\prime}\right)$ which can be embedded in $\mathcal{O}$, which contradicts the maximality of $L$.

Thus, $\bar{L}=K_{1}$.

To prove that $K$ is isomorphic to a skew field $K_{1}\left(\left(t_{2}\right)\right)$ where $t_{2} a=\alpha(a) t_{2}$ one can apply similar arguments as in the proof of the existence of an element $a^{\prime}$ such that $a^{\prime} x a^{\prime-1}=x$ (see above). So, one can find a parameter $t_{2}$ with a given property.

In some cases we have a complete classification of local skew fields.

Proposition ([Zh]). Assume that $K_{1}$ is isomorphic to $k\left(\left(t_{1}\right)\right)$. Put

$$
\zeta=\alpha\left(t_{1}\right) t_{1}^{-1} \bmod \mathcal{M}_{K_{1}} \text {. }
$$

Put $i_{\alpha}=1$ if $\zeta$ is not a root of unity in $k$ and $i_{\alpha}=v_{K_{1}}\left(\alpha^{n}\left(t_{1}\right)-t_{1}\right)$ if $\zeta$ is a primitive $n$th root. Assume that $k$ is of characteristic zero. Then there is an automorphism $f \in \operatorname{Aut}_{k}\left(K_{1}\right)$ such that $f^{-1} \alpha f=\beta$ where

$$
\beta\left(t_{1}\right)=\zeta t_{1}+x t_{1}^{i_{\alpha}}+x^{2} y t_{1}^{2 i_{\alpha}-1}
$$

for some $x \in k^{*} / k^{*\left(i_{\alpha}-1\right)}, y \in k$.

Two automorphisms $\alpha$ and $\beta$ are conjugate if and only if

$$
\left(\zeta(\alpha), i_{\alpha}, x(\alpha), y(\alpha)\right)=\left(\zeta(\beta), i_{\beta}, x(\beta), y(\beta)\right) .
$$

Proof. First we prove that $\alpha=f \beta^{\prime} f^{-1}$ where

$$
\beta^{\prime}\left(t_{1}\right)=\zeta t_{1}+x t_{1}^{i n+1}+y t_{1}^{2 i n+1}
$$

for some natural $i$. Then we prove that $i_{\alpha}=i_{\beta^{\prime}}$.

Consider a set $\left\{\alpha_{i}: i \in \mathbb{N}\right\}$ where $\alpha_{i}=f_{i} \alpha_{i-1} f_{i}^{-1}, f_{i}\left(t_{1}\right)=t_{1}+x_{i} t_{1}^{i}$ for some $x_{i} \in k, \alpha_{1}=\alpha$. Write

$$
\alpha_{i}\left(t_{1}\right)=\zeta t_{1}+a_{2, i} t_{1}^{2}+a_{3, i} t_{1}^{3}+\ldots .
$$


One can check that $a_{2,2}=x_{2}\left(\zeta^{2}-\zeta\right)+a_{2,1}$ and hence there exists an element $x_{2} \in k$ such that $a_{2,2}=0$. Since $a_{j, i+1}=a_{j, i}$, we have $a_{2, j}=0$ for all $j \geqslant 2$. Further, $a_{3,3}=x_{3}\left(\zeta^{3}-\zeta\right)+a_{3,2}$ and hence there exists an element $x_{3} \in k$ such that $a_{3,3}=0$. Then $a_{3, j}=0$ for all $j \geqslant 3$. Thus, any element $a_{k, k}$ can be made equal to zero if $n \nmid(k-1)$, and therefore $\alpha=f \tilde{\alpha} f^{-1}$ where

$$
\tilde{\alpha}\left(t_{1}\right)=\zeta t_{1}+\tilde{a}_{i n+1} t_{1}^{i n+1}+\tilde{a}_{i n+n+1} t_{1}^{i n+n+1}+\ldots
$$

for some $i, \tilde{a}_{j} \in k$. Notice that $\tilde{a}_{i n+1}$ does not depend on $x_{i}$. Put $x=x(\alpha)=\tilde{a}_{i n+1}$.

Now we replace $\alpha$ by $\tilde{\alpha}$. One can check that if $n \mid(k-1)$ then

$$
a_{j, k}=a_{j, k-1} \quad \text { for } 2 \leqslant j<k+i n
$$

and

$a_{k+i n, k}=x_{k} x(k-i n-1)+a_{k+i n}+$ some polynomial which does not depend on $x_{k}$.

From this fact it immediately follows that $a_{2 i n+1, i n+1}$ does not depend on $x_{i}$ and for all $k \neq i n+1 \quad a_{k+i n, k}$ can be made equal to zero. Then $y=y(\alpha)=a_{2 i n+1, i n+1}$.

Now we prove that $i_{\alpha}=i_{\beta^{\prime}}$. Using the formula

$$
\beta^{\prime n}\left(t_{1}\right)=t_{1}+n x(\alpha) \zeta^{-1} t_{1}^{i n+1}+\ldots
$$

we get $i_{\beta^{\prime}}=i n+1$. Then one can check that $v_{K_{1}}\left(f^{-1}\left(\alpha^{n}-\mathrm{id}\right) f\right)=v_{K_{1}}\left(\alpha^{n}-\mathrm{id}\right)=i_{\alpha}$. Since $\beta^{\prime n}-\mathrm{id}=f^{-1}\left(\alpha^{n}-\mathrm{id}\right) f$, we get the identity $i_{\alpha}=i_{\beta^{\prime}}$.

The rest of the proof is clear. For details see [Zh, Lemma 6 and Prop.5].

\subsection{Canonical automorphisms of finite order}

\subsubsection{Characteristic zero case.}

Assume that

a two-dimensional local skew field $K$ splits, $K_{1}$ is a field, $K_{0} \subset Z(K)$, $\operatorname{char}(K)=\operatorname{char}\left(K_{0}\right)=0$, $\alpha^{n}=$ id for some $n \geqslant 1$, for any convergent sequence $\left(a_{j}\right)$ in $K_{1}$ the sequence $\left(t_{2} a_{j} t_{2}^{-1}\right)$ converges in $K$.

Lemma. $K$ is isomorphic to a two-dimensional local skew field $K_{1}\left(\left(t_{2}\right)\right)$ where

$$
t_{2} a t_{2}^{-1}=\alpha(a)+\delta_{i}(a) t_{2}^{i}+\delta_{2 i}(a) t_{2}^{2 i}+\delta_{2 i+n}(a) t_{2}^{2 i+n}+\ldots \quad \text { for all } a \in K_{1}
$$

where $n \mid i$ and $\delta_{j}: K_{1} \rightarrow K_{1}$ are linear maps and

$$
\delta_{i}(a b)=\delta_{i}(a) \alpha(b)+\alpha(a) \delta_{i}(b) \quad \text { for every } a, b \in K_{1} .
$$

Geometry \& Topology Monographs, Volume 3 (2000) - Invitation to higher local fields 
Moreover

$$
t_{2}^{n} a t_{2}^{-n}=a+\delta_{i}^{\prime}(a) t_{2}^{i}+\delta_{2 i}^{\prime}(a) t_{2}^{2 i}+\delta_{2 i+n}^{\prime}(a) t_{2}^{2 i+n}+\ldots
$$

where $\delta_{j}^{\prime}$ are linear maps and $\delta_{i}^{\prime}$ and $\delta:=\delta_{2 i}^{\prime}-((i+1) / 2) \delta_{i}^{\prime 2}$ are derivations.

Remark. The following fact holds for the field $K$ of any characteristic: $K$ is isomorphic to a two-dimensional local skew field $K_{1}\left(\left(t_{2}\right)\right)$ where

$$
t_{2} a t_{2}^{-1}=\alpha(a)+\delta_{i}(a) t_{2}^{i}+\delta_{i+1}(a) t_{2}^{i+1}+\ldots
$$

where $\delta_{j}$ are linear maps which satisfy some identity. For explicit formulas see [Zh, Prop. 2 and Cor.1].

Proof. It is clear that $K$ is isomorphic to a two-dimensional local skew field $K_{1}\left(\left(t_{2}\right)\right)$ where

$$
t_{2} a t_{2}^{-1}=\alpha(a)+\delta_{1}(a) t_{2}+\delta_{2}(a) t_{2}^{2}+\ldots \quad \text { for all } a
$$

and $\delta_{j}$ are linear maps. Then $\delta_{1}$ is a $\left(\alpha^{2}, \alpha\right)$-derivation, that is $\delta_{1}(a b)=\delta_{1}(a) \alpha^{2}(b)+$ $\alpha(a) \delta_{1}(b)$.

Indeed,

$$
\begin{aligned}
& t_{2} a b t_{2}^{-1}=t_{2} a t_{2}^{-1} t_{2} b t_{2}^{-1}=\left(\alpha(a)+\delta_{1}(a) t_{2}+\ldots\right)\left(\alpha(b)+\delta_{1}(b) t_{2}+\ldots\right) \\
= & \alpha(a) \alpha(b)+\left(\delta_{1}(a) \alpha^{2}(b)+\alpha(a) \delta_{1}(b)\right) t_{2}+\ldots=\alpha(a b)+\delta_{1}(a b) t_{2}+\ldots .
\end{aligned}
$$

From the proof of Theorem 8.2 it follows that $\delta_{1}$ is an inner derivation, i.e. $\delta_{1}(a)=$ $g \alpha^{2}(a)-\alpha(a) g$ for some $g \in K_{1}$, and that there exists a $t_{2,2}=\left(1+x_{1} t_{2}\right) t_{2}$ such that

$$
t_{2,2} a t_{2,2}^{-1}=\alpha(a)+\delta_{2,2}(a) t_{2,2}^{2}+\ldots .
$$

One can easily check that $\delta_{2,2}$ is a $\left(\alpha^{3}, \alpha\right)$-derivation. Then it is an inner derivation and there exists $t_{2,3}$ such that

$$
t_{2,3} a t_{2,3}^{-1}=\alpha(a)+\delta_{3,3}(a) t_{2,3}^{3}+\ldots .
$$

By induction one deduces that if

$$
t_{2, j} a t_{2, j}^{-1}=\alpha(a)+\delta_{n, j}(a) t_{2, j}^{n}+\ldots+\delta_{k n, j}(a) t_{2, j}^{k n}+\delta_{j, j}(a) t_{2, j}^{j}+\ldots
$$

then $\delta_{j, j}$ is a $\left(\alpha^{j+1}, \alpha\right)$-derivation and there exists $t_{2, j+1}$ such that

$$
t_{2, j+1} a t_{2, j+1}^{-1}=\alpha(a)+\delta_{n, j}(a) t_{2, j+1}^{n}+\ldots+\delta_{k n, j}(a) t_{2, j+1}^{k n}+\delta_{j+1, j+1}(a) t_{2, j+1}^{j+1}+\ldots
$$

The rest of the proof is clear. For details see [Zh, Prop.2, Cor.1, Lemmas 10, 3].

Definition. Let $i=v_{K_{2}}\left(\varphi\left(t_{2}^{n}\right)\left(t_{1}\right)-t_{1}\right) \in n \mathbb{N} \cup \infty,(\varphi$ is defined in subsection 8.1) and let $r \in \mathbb{Z} / i$ be $v_{K_{1}}(x) \bmod i$ where $x$ is the residue of $\left(\varphi\left(t_{2}^{n}\right)\left(t_{1}\right)-t_{1}\right) t_{2}^{-i}$. Put

$$
a=\operatorname{res}_{t_{1}}\left(\frac{\left(\delta_{2 i}^{\prime}-\frac{i+1}{2} \delta_{i}^{\prime 2}\right)\left(t_{1}\right)}{\delta_{i}^{\prime}\left(t_{1}\right)^{2}} d t_{1}\right) \in K_{0} .
$$

Geometry \& Topology Monographs, Volume 3 (2000) - Invitation to higher local fields 
( $\delta_{i}^{\prime}, \delta_{2 i}^{\prime}$ are the maps from the preceding lemma).

Proposition. If $n=1$ then $i, r$ don't depend on the choice of a system of local parameters; if $i=1$ then a does not depend on the choice of a system of local parameters; if $n \neq 1$ then a depends only on the maps $\delta_{i+1}, \ldots, \delta_{2 i-1}, i, r$ depend only on the maps $\delta_{j}, j \notin n \mathbb{N}, j<i$.

Proof. We comment on the statement first. The maps $\delta_{j}$ are uniquely defined by parameters $t_{1}, t_{2}$ and they depend on the choice of these parameters. So the claim that $i, r$ depend only on the maps $\delta_{j}, j \notin n \mathbb{N}, j<i$ means that $i, r$ don't depend on the choice of parameters $t_{1}, t_{2}$ which preserve the maps $\delta_{j}, j \notin n \mathbb{N}, j<i$.

Note that $r$ depends only on $i$. Hence it is sufficient to prove the proposition only for $i$ and $a$. Moreover it suffices to prove it for the case where $n \neq 1, i \neq 1$, because if $n=1$ then the sets $\left\{\delta_{j}: j \notin n \mathbb{N}\right\}$ and $\left\{\delta_{i+1}: \ldots, \delta_{2 i-1}\right\}$ are empty.

It is clear that $i$ depends on $\delta_{j}, j \notin n \mathbb{N}$. Indeed, it is known that $\delta_{1}$ is an inner $\left(\alpha^{2}, \alpha\right)$-derivation (see the proof of the lemma). By [Zh, Lemma 3] we can change a parameter $t_{2}$ such that $\delta_{1}$ can be made equal $\delta_{1}\left(t_{1}\right)=t_{1}$. Then one can see that $i=1$. From the other hand we can change a parameter $t_{2}$ such that $\delta_{1}$ can be made equal to 0 . In this case $i>1$. This means that $i$ depends on $\delta_{1}$. By [Zh, Cor.3] any map $\delta_{j}$ is uniquely determined by the maps $\delta_{q}, q<j$ and by an element $\delta_{j}\left(t_{1}\right)$. Then using similar arguments and induction one deduces that $i$ depends on other maps $\delta_{j}$, $j \notin n \mathbb{N}, j<i$.

Now we prove that $i$ does not depend on the choice of parameters $t_{1}, t_{2}$ which preserve the maps $\delta_{j}, j \notin n \mathbb{N}, j<i$.

Note that $i$ does not depend on the choice of $t_{1}$ : indeed, if $t_{1}^{\prime}=t_{1}+b z^{j}, b \in K_{1}$ then $z^{n} t_{1}^{\prime} z^{-n}=z^{n} t_{1} z^{-n}+\left(z^{n} b z^{-n}\right) z^{j}=t_{1}^{\prime}+r$, where $r \in \mathcal{M}_{K}^{i} \backslash \mathcal{M} \mathbb{N}_{K}^{i+1}$. One can see that the same is true for $t_{1}^{\prime}=c_{1} t_{1}+c_{2} t_{2}^{2}+\ldots, c_{j} \in K_{0}$.

Let $\delta_{q}$ be the first non-zero map for given $t_{1}, t_{2}$. If $q \neq i$ then by [Zh, Lemma 8 , (ii)] there exists a parameter $t_{1}^{\prime}$ such that $z t_{1}^{\prime} z^{-1}=t_{1}^{\prime \alpha}+\delta_{q+1}\left(t_{1}^{\prime}\right) z^{q+1}+\ldots$ Using this fact and Proposition 8.2 we can reduce the proof to the case where $q=i, \alpha\left(t_{1}\right)=\xi t_{1}$, $\alpha\left(\delta_{i}\left(t_{1}\right)\right)=\xi \delta_{i}\left(t_{1}\right)$ (this case is equivalent to the case of $\left.n=1\right)$. Then we apply [Zh, Lemma 3] to show that

$$
v_{K_{2}}\left(\left(\phi\left(t_{2}^{\prime}\right)-1\right)\left(t_{1}\right)\right)=v_{K_{2}}\left(\left(\phi\left(t_{2}\right)-1\right)\left(t_{1}\right)\right),
$$

for any parameters $t_{2}, t_{2}^{\prime}$, i.e. $i$ does not depend on the choice of a parameter $t_{2}$. For details see [Zh, Prop.6].

To prove that $a$ depends only on $\delta_{i+1}, \ldots, \delta_{2 i-1}$ we use the fact that for any pair of parameters $t_{1}^{\prime}, t_{2}^{\prime}$ we can find parameters $t_{1}^{\prime \prime}=t_{1}+r$, where $r \in \mathcal{M}_{K}^{i}, t_{2}^{\prime \prime}$ such that corresponding maps $\delta_{j}$ are equal for all $j$. Then by [Zh, Lemma 8] $a$ does not depend on $t_{1}^{\prime \prime}$ and by [Zh, Lemma 3] $a$ depends on $t_{2}^{\prime \prime}=t_{2}+a_{1} t_{2}^{2}+\ldots, a_{j} \in K_{1}$ if and only if $a_{1}=\ldots=a_{i-1}$. Using direct calculations one can check that $a$ doesn't depend on $t_{2}^{\prime \prime}=a_{0} t_{2}, a_{0} \in K_{1}^{*}$. 
To prove the fact it is sufficient to prove it for $t_{1}^{\prime \prime}=t_{1}+c t_{1}^{h} z^{j}$ for any $j<i, c \in K_{0}$. Using [Zh, Lemma 8] one can reduce the proof to the assertion that some identity holds. The identity is, in fact, some equation on residue elements. One can check it by direct calculations. For details see [Zh, Prop.7].

Remark. The numbers $i, r, a$ can be defined only for local skew fields which splits. One can check that the definition can not be extended to the skew field in Dubrovin's example.

\section{Theorem.}

(1) $K$ is isomorphic to a two-dimensional local skew field $K_{0}\left(\left(t_{1}\right)\right)\left(\left(t_{2}\right)\right)$ such that

$$
t_{2} t_{1} t_{2}^{-1}=\xi t_{1}+x t_{2}^{i}+y t_{2}^{2 i}
$$

where $\xi$ is a primitive $n$th root, $x=c t_{1}^{r}, c \in K_{0}^{*} /\left(K_{0}^{*}\right)^{d}$,

$$
y=(a+r(i+1) / 2) t_{1}^{-1} x^{2}, \quad d=\operatorname{gcd}(r-1, i) .
$$

If $n=1, i=\infty$, then $K$ is a field.

(2) Let $K, K^{\prime}$ be two-dimensional local skew fields of characteristic zero which splits; and let $K_{1}, K_{1}^{\prime}$ be fields. Let $\alpha^{n}=\mathrm{id}, \alpha^{n^{\prime}}=\mathrm{id}$ for some $n, n^{\prime} \geqslant 1$. Then $K$ is isomorphic to $K^{\prime}$ if and only if $K_{0}$ is isomorphic to $K_{0}^{\prime}$ and the ordered sets $(n, \xi, i, r, c, a)$ and $\left(n^{\prime}, \xi^{\prime}, i^{\prime}, r^{\prime}, c^{\prime}, a^{\prime}\right)$ coincide.

Proof. (2) follows from the Proposition of 8.2 and (1). We sketch the proof of (1).

From Proposition 8.2 it follows that there exists $t_{1}$ such that $\alpha\left(t_{1}\right)=\xi t_{1} ; \delta_{i}\left(t_{1}\right)$ can be represented as $c t_{1}^{r} a^{i}$. Hence there exists $t_{2}$ such that

$$
t_{2} t_{1} t_{2}^{-1}=\xi t_{1}+x t_{2}^{i}+\delta_{2 i}\left(t_{1}\right) t_{2}^{2 i}+\ldots
$$

Using [Zh, Lemma 8] we can find a parameter $t_{1}^{\prime}=t_{1} \bmod \mathcal{M}_{K}$ such that

$$
t_{2} t_{1}^{\prime} t_{2}^{-1}=\xi t_{1}+x t_{2}^{i}+y t_{2}^{2 i}+\ldots
$$

The rest of the proof is similar to the proof of the lemma. Using [Zh, Lemma 3] one can find a parameter $t_{2}^{\prime}=t_{2} \bmod \mathcal{M}_{K}^{2}$ such that $\delta_{j}\left(t_{1}\right)=0, j>2 i$.

Corollary. Every two-dimensional local skew field $K$ with the ordered set

$$
(n, \xi, i, r, c, a)
$$

is a finite-dimensional extension of a skew field with the ordered set $(1,1,1,0,1, a)$.

Remark. There is a construction of a two-dimensional local skew field with a given set $(n, \xi, i, r, c, a)$. 


\section{Examples.}

(1) The ring of formal pseudo-differential equations is the skew field with the set $(n=1, \xi=1, i=1, r=0, c=1, a=0)$.

(2) The elements of $\operatorname{Br}(L)$ where $L$ is a two-dimensional local field of equal characteristic are local skew fields. If, for example, $L$ is a $C_{2}$ - field, they split and $i=\infty$. Hence any division algebra in $\operatorname{Br}(L)$ is cyclic.

\subsubsection{Characteristic $p$ case.}

Theorem. Suppose that a two-dimensional local skew field $K$ splits, $K_{1}$ is a field, $K_{0} \subset Z(K)$, $\operatorname{char}(K)=\operatorname{char}\left(K_{0}\right)=p>2$ and $\alpha=\mathrm{id}$.

Then $K$ is a finite dimensional vector space over its center if and only if $K$ is isomorphic to a two-dimensional local skew field $K_{0}\left(\left(t_{1}\right)\right)\left(\left(t_{2}\right)\right)$ where

$$
t_{2}^{-1} t_{1} t_{2}=t_{1}+x t_{2}^{i}
$$

with $x \in K_{1}^{p},(i, p)=1$.

Proof. The "if" part is obvious. We sketch the proof of the "only if" part.

If $K$ is a finite dimensional vector space over its center then $K$ is a division algebra over a henselian field. In fact, the center of $K$ is a two-dimensional local field $k((u))((t))$. Then by [JW, Prop.1.7] $K_{1} /(Z(K))_{1}$ is a purely inseparable extension. Hence there exists $t_{1}$ such that $t_{1}^{p^{k}} \in Z(K)$ for some $k \in \mathbb{N}$ and $K \simeq K_{0}\left(\left(t_{1}\right)\right)\left(\left(t_{2}\right)\right)$ as a vector space with the relation

$$
t_{2} t_{1} t_{2}^{-1}=t_{1}+\delta_{i}\left(t_{1}\right) t_{2}^{i}+\ldots
$$

(see Remark 8.3.1). Then it is sufficient to show that $i$ is prime to $p$ and there exist parameters $t_{1} \in K_{1}, t_{2}$ such that the maps $\delta_{j}$ satisfy the following property:

(*) If $j$ is not divisible by $i$ then $\delta_{j}=0$. If $j$ is divisible by $i$ then $\delta_{j}=c_{j / i} \delta_{i}^{j / i}$ with some $c_{j / i} \in K_{1}$.

Indeed, if this property holds then by induction one deduces that $c_{j / i} \in K_{0}$, $c_{j / i}=((i+1) \ldots(i(j / i-1)+1)) /(j / i) !$. Then one can find a parameter $t_{2}^{\prime}=b t_{2}$, $b \in K_{1}$ such that $\delta_{j}^{\prime}$ satisfies the same property and $\delta_{i}^{2}=0$. Then

$$
t_{2}^{\prime-1} t_{1} t_{2}^{\prime}=t_{1}-\delta_{i}^{\prime}\left(t_{1}\right) t_{2}^{i}
$$

First we prove that $(i, p)=1$. To show it we prove that if $p \mid i$ then there exists a map $\delta_{j}$ such that $\delta_{j}\left(t_{1}^{p^{k}}\right) \neq 0$. To find this map one can use [Zh, Cor.1] to show that $\delta_{i p}\left(t_{1}^{p}\right) \neq 0, \delta_{i p^{2}}\left(t_{1}^{p^{2}}\right) \neq 0, \ldots, \delta_{i p^{k}}\left(t_{1}^{p^{k}}\right) \neq 0$.

Then we prove that for some $t_{2}$ property $(*)$ holds. To show it we prove that if property $(*)$ does not hold then there exists a map $\delta_{j}$ such that $\delta_{j}\left(t_{1}^{p^{k}}\right) \neq 0$. To find this map we reduce the proof to the case of $i \equiv 1 \bmod p$. Then we apply the following idea. 
Let $j \equiv 1 \bmod p$ be the minimal positive integer such that $\delta_{j}$ is not equal to zero on $K_{1}^{p^{l}}$. Then one can prove that the maps $\delta_{m}, k j \leqslant m<(k+1) j, k \in\{1, \ldots, p-1\}$ satisfy the following property:

there exist elements $c_{m, k} \in K_{1}$ such that

$$
\left.\left(\delta_{m}-c_{m, 1} \delta-\ldots-c_{m, k} \delta^{k}\right)\right|_{K_{1}^{p^{l}}}=0
$$

where $\delta: K_{1} \rightarrow K_{1}$ is a linear map, $\left.\delta\right|_{K_{1}^{p^{l}}}$ is a derivation, $\delta\left(t_{1}^{j}\right)=0$ for $j \notin p^{l} \mathbb{N}$, $\delta\left(t_{1}^{p^{l}}\right)=1, c_{k j, k}=c\left(\delta_{j}\left(t_{1}^{p^{l}}\right)\right)^{k}, c \in K_{0}$.

Now consider maps $\widetilde{\delta_{q}}$ which are defined by the following formula

$$
t_{2}^{-1} a t_{2}=a+\widetilde{\delta_{i}}(a) t_{2}^{i}+\widetilde{\delta_{i+1}}(a) t_{2}^{i+1}+\ldots, \quad a \in K_{1} .
$$

Then $\widetilde{\delta_{q}}+\delta_{q}+\sum_{k=1}^{q-1} \delta_{k} \widetilde{\delta_{q-k}}=0$ for any $q$. In fact, $\widetilde{\delta_{q}}$ satisfy some identity which is similar to the identity in [Zh, Cor.1]. Using that identity one can deduce that if

$j \equiv 1 \bmod p$ and there exists the minimal $m(m \in \mathbb{Z})$ such that $\left.\delta_{m p+2 i}\right|_{K_{1}^{p^{l}}} \neq 0$ if $j \chi(m p+2 i)$ and $\left.\delta_{m p+2 i}\right|_{K_{1}^{p^{l}}} \neq\left. s \delta_{j}^{(2 i+m p) / j}\right|_{K_{1}^{p^{l}}}$ for any $s \in K_{1}$ otherwise, and $\delta_{q}\left(t_{1}^{p^{l}}\right)=0$ for $q<m p+2 i, q \not \equiv 1 \bmod p$, then

$(m p+2 i)+(p-1) j$ is the minimal integer such that $\left.\delta_{(m p+2 i)+(p-1) j}\right|_{K_{1}^{p l+1}} \neq 0$.

To complete the proof we use induction and [Zh, Lemma 3] to show that there exist parameters $t_{1} \in K_{1}, t_{2}$ such that $\delta_{q}\left(t_{1}^{p^{l}}\right)=0$ for $q \not \equiv 1,2 \bmod p$ and $\delta_{j}^{2}=0$ on $K_{1}^{p^{l}}$.

Corollary 1. If $K$ is a finite dimensional division algebra over its center then its index is equal to $p$.

Corollary 2. Suppose that a two-dimensional local skew field $K$ splits, $K_{1}$ is a field, $K_{0} \subset Z(K)$, char $(K)=\operatorname{char}\left(K_{0}\right)=p>2, K$ is a finite dimensional division algebra over its center of index $p^{k}$.

Then either $K$ is a cyclic division algebra or has index $p$.

Proof. By [JW, Prop. 1.7] $K_{1} / \overline{Z(K)}$ is the compositum of a purely inseparable extension and a cyclic Galois extension. Then the canonical automorphism $\alpha$ has order $p^{l}$ for some $l \in \mathbb{N}$. By [Zh, Lemma 10] (which is true also for $\operatorname{char}(K)=p>0$ ), $K \simeq K_{0}\left(\left(t_{1}\right)\right)\left(\left(t_{2}\right)\right)$ with

$$
t_{2} a t_{2}^{-1}=\alpha(a)+\delta_{i}(a) t_{2}^{i}+\delta_{i+p^{l}}(a) t_{2}^{i+p^{l}}+\delta_{i+p^{l}}(a) t_{2}^{i+2 p^{l}}+\ldots
$$


where $i \in p^{l} \mathbb{N}, a \in K_{1}$. Suppose that $\alpha \neq 1$ and $K_{1}$ is not a cyclic extension of $\overline{Z(K)}$. Then there exists a field $F \subset K_{1}, F \not \subset Z(K)$ such that $\left.\alpha\right|_{F}=1$. If $a \in F$ then for some $m$ the element $a^{p^{m}}$ belongs to a cyclic extension of the field $\overline{Z(K)}$, hence $\delta_{j}\left(a^{p^{m}}\right)=0$ for all $j$. But we can apply the same arguments as in the proof of the preceding theorem to show that if $\delta_{i} \neq 0$ then there exists a map $\delta_{j}$ such that $\delta_{j}\left(a^{p^{m}}\right) \neq 0$, a contradiction. We only need to apply [Zh, Prop.2] instead of [Zh, Cor.1] and note that $\alpha \delta=x \delta \alpha$ where $\delta$ is a derivation on $K_{1}, x \in K_{1}, x \equiv 1 \bmod \mathcal{M}_{K_{1}}$, because $\alpha\left(t_{1}\right) / t_{1} \equiv 1 \bmod \mathcal{M}_{K_{1}}$.

Hence $t_{2} a t_{2}^{-1}=\alpha(a)$ and $K_{1} / \overline{Z(K)}$ is a cyclic extension and $K$ is a cyclic division algebra $\left(K_{1}\left(t_{2}^{p^{k}}\right) / Z(K), \alpha, t_{2}^{p^{k}}\right)$.

Corollary 3. Let $F=F_{0}\left(\left(t_{1}\right)\right)\left(\left(t_{2}\right)\right)$ be a two-dimensional local field, where $F_{0}$ is an algebraically closed field. Let $A$ be a division algebra over $F$.

Then $A \simeq B \otimes C$, where $B$ is a cyclic division algebra of index prime to $p$ and $C$ is either cyclic (as in Corollary 2) or $C$ is a local skew field from the theorem of index $p$.

Proof. Note that $F$ is a $C_{2}$-field. Then $A_{1}$ is a field, $A_{1} / F_{1}$ is the compositum of a purely inseparable extension and a cyclic Galois extension, and $A_{1}=F_{0}((u))$ for some $u \in A_{1}$. Hence $A$ splits. So, $A$ is a splitting two-dimensional local skew field.

It is easy to see that the index of $A$ is $|\bar{A}: \bar{F}|=p^{q} m,(m, p)=1$. Consider subalgebras $B=C_{A}\left(F_{1}\right), C=C_{A}\left(F_{2}\right)$ where $F_{1}=F\left(u^{p^{q}}\right), F_{2}=F\left(u^{m}\right)$. Then by [M, Th.1] $A \simeq B \otimes C$.

The rest of the proof is clear.

Now one can easily deduce that

Corollary 4. The following conjecture: the exponent of $A$ is equal to its index for any division algebra $A$ over a $C_{2}$-field $F$ (see for example [PY, 3.4.5.]) has the positive answer for $F=F_{0}\left(\left(t_{1}\right)\right)\left(\left(t_{2}\right)\right)$.

\section{Reference}

[JW] B. Jacob and A. Wadsworth, Division algebras over Henselian fields, J.Algebra, 128(1990), p. 126-179.

[M] P. Morandi, Henselisation of a valued division algebra, J. Algebra, 122(1989), p.232-243.

[Pa] A.N. Parshin, On a ring of formal pseudo-differential operators, Proc. Steklov Math. Inst., v.224, 1999, pp. 266-280, (alg-geom/ 9911098). 
[PY] V.P. Platonov and V.I. Yanchevskii, Finite dimensional division algebras, VINITY, 77(1991), p.144-262 (in Russian)

[Zh] A. B. Zheglov, On the structure of two-dimensional local skew fields, to appear in Izv. RAN Math. (2000).

Department of Algebra, Steklov Institute, Ul. Gubkina, 8, Moscow GSP-1, 117966 Russia. E-mail: abzv24@mail.ru,azheglov@chat.ru 